\title{
Quasi 3-D Simulation of Quantum Well DFB Lasers
}

\author{
B. Witzigmann ${ }^{a}$, F. Oyafuso ${ }^{b}$ and K. Hess ${ }^{b}$ \\ antegrated Systems Laboratory, ETH Zürich \\ Gloriastr. $92 \mathrm{CH}-8092$ Zürich, Switzerland \\ ${ }^{b}$ Beckman Institute for Advanced Science and Technology \\ 405 North Mathews Avenue, Urbana IL 61801, USA
}

\begin{abstract}
A quasi-3 D simulation for quantum well (QW) distributed feedback (DFB) laser diodes is presented. Longitudinal effects such as the nonuniformity of the optical intensity, local gain and spontaneous emission are considered as well as lateral effects such as current spreading and quantum carrier capture. The simulation is performed by solving several 2-D cross-sections using Minilase II and a 1-D longitudinal solver in a self-consistent fashion.

As an example an analysis of spatial hole burning in a $\lambda / 4$ phase shifted DFB laser is given. It is shown that spatial hole burning (SHB) results in a local gain saturation and is mainly restricted to electrons and holes within the QW.
\end{abstract}

\section{Introduction}

Quantum well distributed feedback lasers (DFB) have been simulated mostly by one dimensional models which account for the fact that the longitudinal optical and electronic properties change along the cavity axis [1] [2]. The simulation of such a device must account for this and other physical aspects. The optical intensity varies along this axis and therefore influences electronic propertics such as carrier and current distribution, stimulated and spontancous emission and most importantly the singlemode stability of the DFB laser.

A more rigorous description of the electronic properties needs to include electron and hole dynamics in both the bulk and in the quantum well. Lateral current spreading is of significance for the confinement and optimization of the optical intensity. These physical properties have been studied in 2-D simulations of cross-sections of Fabry Perot lasers using Minilase II [3].

In this paper, a quasi-3-D simulation of a quantum well DFB laser is presented that accounts both for the longitudinal and for the lateral effects. The simulation consists of a $1-D$ portion that links several 2-D cross-sections. The 2-D calculations are performed with the quantum well laser simulator Minilase II. 


\section{Theory}

We consider a laser waveguide that may have an arbitrary type of grating corrugation and that consists of several sections which contain phaseshifts or passive regions (multisection cavity).

Following the approach in [4] the laser cavity is treated as open resonator with a Langevin noise source that accounts for the spontaneous emission. The fundamental transverse mode is obtained by solving the scalar Helmholtz equation of a crosssection of the device. The calculation of the longitudinal mode includes phase-shifts, passive sections and different facet reflectivities.

The calculation of the electronic properties of the DFB laser is based on the 2dimensional laser simulator Minilase II. In Minilase II, the solution of the two dimensional electronic equations that determine electron and hole supply to the active lasing region is accomplished along the lines of silicon device simulation [5] with the addition of specific provisions that describe transport over abrupt heterojunctions [6] and capture in the active quantum well region. Within the quantum wells we solve a Boltzmann transport equation in energy space and a rate equation for phonon heating. The resulting electrical simulator has been described in detail in [3] and represents the core of the Minilase II simulation package.

The link between the 1-dimensional longitudinal solver and the 2-dimensional simulation of Minilase II is realized mainly by the modal photon rate equation where the spontancous and stimulated emission coefficients are weighted by the spatial optical distribution and integrated over the cavity volume.

The basic idea of the simulation procedure is to divide the cavity in a number of cross-sections parallel to the facets. For each cross-section, Minilase II solves the electronic equations subject to the constraint that the total photon population over all cross-sections is held constant. After one iteration the photon number is updated by solving the photon rate equation.

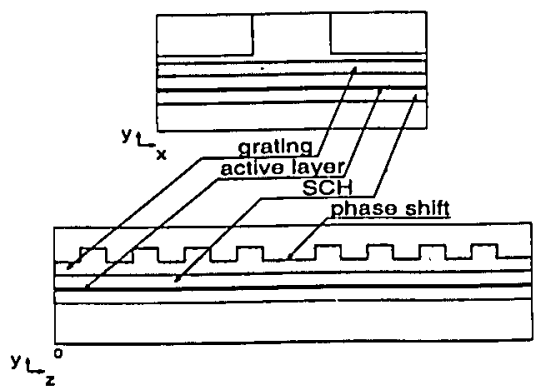

Figure 1: A schematic representation of the ridge-waveguide DFB laser simulated in the paper.

\section{Results}

As an example spatial hole burning in a $\lambda / 4$ phase shifted DFB laser is analyzed. It is a ridge waveguide $Q W$ laser at the wavelength $980 \mathrm{~nm}$. The length of the laser cavity is $400 \mu \mathrm{m}$ and the coupling coefficient $\kappa l$ is 1.7 , the reflectivity of the facets is 0 and 

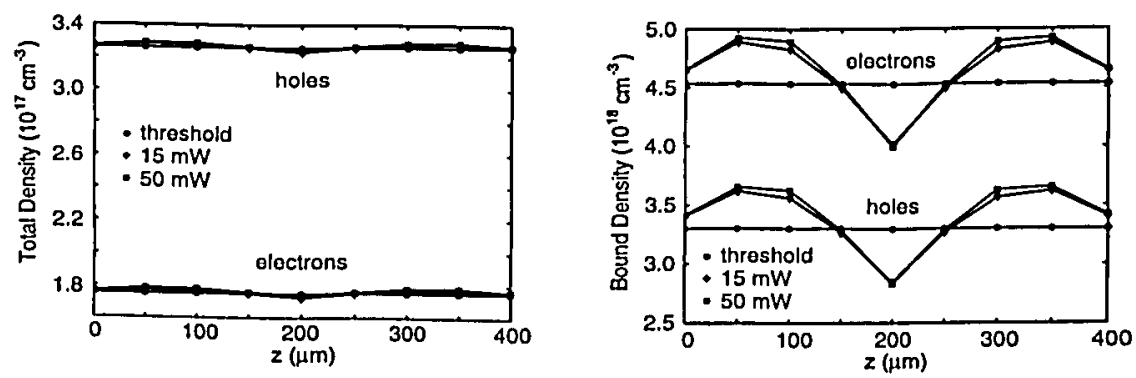

Figure 2: Total carrier density (a) and bound quantum carrier density (b) along the cavity axis. The densities are averaged over the corresponding xy-cross-section.

the $\lambda / 4$ phase shift is located in the middle of the cavity. A schematic representation of the geometry is illustrated in Figure 1.

Quasi-3-dimensional simulations are carried out at 3 biaspoints, at threshold, at an output power of $15 \mathrm{~mW}$ and at an output power of $50 \mathrm{~mW}$. We focus on the physical properties in the longitudinal direction. Therefore, all the carrier concentrations shown in the illustrations are averaged over a cross-section of the device. Figure 2 a. shows the total concentration of electrons and holes which consists of both carriers in the bulk and the bound and continuum of carriers in the quantum well. At threshold the carrier concentration of both holes and the electrons is constant in space. At higher power levels spatial hole burning becomes visible. The total concentrations vary only by about $1 \%$ in the longitudinal direction. As a consequence spatial hole burning in the quantum well has nearly no effect on the bulk carrier concentration. In contrast, strong SHB $(\approx 25 \%)$ can be seen in the concentration of the bound carriers in the quantum well states which is illustrated in Figure $2 \mathrm{~b}$. Spatial hole burning is thus localized in the quantum-well where the electron photon interaction takes place. Looking at local gain along the $\mathrm{z}$-axis, the simulations show that with increasing power, local gain saturates to the loss of the cavity (see fig. 3). With increasing output power, local gain decreases in the phaseshift region and increases in the side regions along the cavity axis. This modification also happens to the carrier densities.

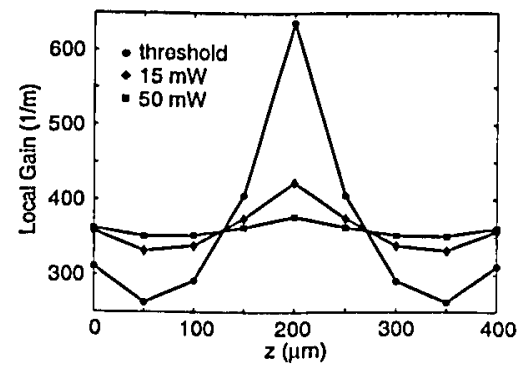

Figure 3: Local gain at 3 different output powers for a $\lambda / 4$ phase shifted $Q W-D F B$ laser $(\kappa l=1.7)$. 


\section{Conclusion}

A quasi-3-dimensional simulation for QW DFB lasers has been presented. For the longitudinal properties the transfer matrix method has been used. For the lateral properties, the QW laser simulator Minilase II has calculated several cross-sections. It includes the ballistic injection of bulk carriers into the quantum well, the scattering dynamics of quantum carriers and quantum carrier temperature.

The simulator has then been used to investigate the spatial hole burning effect of a $\lambda / 4$ phase shifted QW DFB laser. It has been shown that spatial hole burning results in a saturation of local gain approaching the loss and is mainly restricted to electrons and holes within the $\mathrm{QW}$ region.

\section{Acknowledgements}

The authors would like to thank Prof. W. Fichtner (ETH Zurich) for his contributions. K. Hess and F. Oyafuso were supported by the Office of Naval Research and by the National Science Foundation through NCCE. The authors also would like to thank M. Grupen for his contributions to this work.

\section{References}

[1] T. Makino, "Amplified Spontaneous Emission Model for Quantum-Well Distributed Feedback Lasers", Journal of Quantum Electronics, June 1997 Vol. 33, No 6, pg. $1010-1017$.

[2] B. Tromberg, H. Olesen, X. Pan, Theory of Linewidth for Multielectrode Laser Diodes with Spatially Distributed Noise Sources, IEEE Journal of Quantum ELectronics, February 1991 Vol. 27, No.2, pg. 178-192.

[3] M. Grupen, K. Hess, "Simulation of Carrier Transport and Nonlinearities in Quantum Well Laser Diodes", IEEE Journal of Quantum Electronics, January 1998, Vol. 34, No. 1, pg. 120-140.

[4] C.H. Henry, Theory of Spontaneous Emission Noise in Open Resonators and its Application to Lasers and Optical Amplifiers, Journal of Lightwave Technology, March 1986, Vol. LT -4 , No. 3, pg. 288-297.

[5] S. Selberherr, "Analysis and Simulation of Semiconductor Devices", Springer Verlag, Wien-New York, 1984.

[6] M. Grupen, K. Hess, and G.H. Song, "Simulation of transport over heterojunctions", Proc. 4th International Conf. Simul. Semicon. Dev. Process., Zurich, vol 4, 303-311 (1991). 